\title{
Estudo e aplicação de quatérnios em processamento digital de imagens
}

\author{
Diego Ponciano de Oliveira Lima \\ Docente. Instituto Federal de Educação, Ciência e Tecnologia do Ceará (IFCE) \\ (poncianodiego@gmail.com)
}

\begin{abstract}
Resumo: A transformada de Fourier é utilizada para decompor uma imagem nas suas componentes de frequência para vários tipos de processamento digital de imagens como filtragem, compressão e restauração. Os métodos de processamento de imagem proporcionam grandes avanços em diversas áreas de conhecimento, como na Medicina, em que diagnósticos médicos são feitos com a análise de imagens que necessitam de tratamento, como a filtragem. Em imagens coloridas de marca d'água, são necessários tratamentos com filtros de modo que a imagem não tenha ruídos ou até mesmo a compressão dessas imagens, de tal modo que quando houver uma descompressão não haja erros. Hoje, técnicas de filtragem de imagem colorida são representadas por rotações no espaço de cores tridimensional. Tais rotações são facilmente representadas pela álgebra dos Quatérnios de Hamilton. O presente trabalho analisa as várias aplicações da transformada quaterniônica de Fourier no processamento de imagens, mostrando a eficácia da transformada quaterniônica de Fourier comparada com a transformada de Fourier convencional.
\end{abstract}

Palavras-chave: Quatérnios; Transformada de Fourier; Processamento Digital de Imagens.

\section{Study and application of quaternions in digital image processing}

\begin{abstract}
The Fourier transform is used to decompose an image into its frequency components for various types of digital image processing such as filtering, compression and restoration. Image processing methods provide breakthroughs in a variety of areas of expertise, such as in medical imaging where medical diagnostics are done by analyzing images that require treatment such as filtering. In color watermarked images, filters treatments are required so that the image does not have noises or even the compression of such images so that when there is a decompression there are no errors. Nowadays, color image filtering techniques are represented by rotations in three-dimensional color space, such rotations are easily represented by Hamilton's Quanternion Algebra. The present work analyses the various applications of the quaternion Fourier transform in the image processing showing the effectiveness of the quaternion Fourier transform compared to the conventional Fourier transform.
\end{abstract}

Keywords: Quaternion; Fourier transform; Digital image processing.

\section{INTRODUÇÃO}

A transformada de Fourier tem aplicações em diversas áreas de conhecimento, como Física, Teoria dos Números, Análise Combinatória, Processamento de Sinais e Processamento de Imagens, entre outras. Nos campos relacionados com processamento de sinais e de imagens, a transformada de Fourier é tipicamente utilizada para decompor um sinal nas suas componentes em frequência e suas amplitudes para vários tipos processamento como filtragem, compressão e restauração. Os métodos de processamento de imagem proporcionam grandes avanços em diversas áreas de conhecimento, como na 
Medicina, em que diagnósticos médicos são feitos com a análise de imagens que necessitam do tratamento como a filtragem.

Em imagens coloridas de marca d'água, são necessários tratamentos com filtro passa - baixa ou passa - alta de modo que a imagem não tenha ruídos ou até mesmo a compressão dessas imagens de tal modo que quando houver uma descompressão não haja erros (SANGWINE, 1997).

Segundo Neto (1999), um dos processos mais utilizados no trabalho de processamento de sinais e imagens é a filtragem no qual é utilizada a transformada de Fourier, pois filtrar um sinal nada mais é que multiplicar uma função filtro no domínio de Fourier pelo sinal também no domínio de Fourier. Considerando sinais em duas dimensões (ou imagens) é usada a transformada de Fourier bidimensional para efetuar a filtragem.

Até recentemente, nenhum texto matemático tinha sugerido aplicações a filtragem de imagens coloridas. Hoje é possível desenvolver filtros baseados no espaço de cores como o espaço RGB (SANGWINE; ELL, 2000).

Matematicamente, rotações em espaços tridimensionais (por exemplo, o espaço de cores RGB) podem ser representadas pela Álgebra dos Quatérnios. Neste contexto, é possível aplicar essa ferramenta mais conhecida como números Hipercomplexos e juntamente com a clássica Transformada de Fourier desenvolvendo, um caso particular das transformadas hipercomplexas, a Transformada Quaterniônica de Fourier.

Para Sangwine (2008), a principal motivação para a utilização da álgebra dos Quatérnios para lidar com tais sinais é a correspondência entre as amostras de um dado vetor e os elementos das partes vetoriais do quatérnio, que permite o processamento holístico das amostras de vetores e de sinais, fazendo uso de informações sobre a direção e magnitude das amostras no espaço amostral.

A primeira aplicação da Transformada Quaterniônica de Fourier em imagem colorida foi relatada em 1996 por Sangwine, usando a versão discreta da Transformada definida por Ell. Estas definições das Transformadas hipercomplexas de Fourier têm demonstrado a sua aplicabilidade, permitindo generalizações de técnicas de processamento de imagens (SANGWINE; ELL, 2007).

A necessidade de utilizar a transformada de Fourier não é trivialmente aparente, mas muitas operações requerem a utilização de tal ferramenta, como a filtragem linear com máscaras de convolução muito grande que podem ser realizadas com 
sucesso usando componentes de cor separados. Isso é possível, pois as operações de filtragem não dependem da correlação entre as três componentes de cor (Ell and Sangwine, 2007). Neste sentido, o presente artigo avalia algumas aplicações da transformada quaterniônica de Fourier nas operações processamento de imagens como restauração e filtragem.

\section{FUNDAMENTAÇÃO TEÓRICA}

Para Neto (1999), o interesse em métodos de processamento de sinais e imagens está principalmente em duas áreas de aplicações: a melhoria de informação (sinal ou imagem) para interpretação humana, e processamento de dados (sinal ou imagem) em computador, e vem crescendo com aplicações no Programa Espacial, na Medicina, Física, Indústria, etc. Segundo Gonzalez (1993), o termo imagem refere-se a uma função de intensidade de luz bidimensional $f(x, y)$, onde $x$ e $y$ são coordenadas espaciais e o valor de $f$ em um ponto qualquer $(x, y)$ é proporcional ao brilho ou nível de cinza da imagem naquele ponto. Uma imagem digital é uma imagem $f(x, y)$ discretizada no espaço e na intensidade de brilho e pode ser considerada uma matriz, cujos elementos são chamados e pixels.

A transformada de Fourier e a Transformada Discreta de Fourier (unidimensional ou bidimensional) são ferramentas matemáticas de grande aplicabilidade na solução dos problemas de processamento de imagens (sinais bidimensionais), pois muitas vezes, é conveniente a mudança do domínio do tempo ou espaço $(x, y)$ para o domínio da frequência para conhecer as componentes de frequência presentes de um dado sinal. Na prática, quando se quer trabalhar uma imagem no domínio da frequência, por exemplo, simplesmente efetua a Transformada de Fourier da referida imagem e a multiplica pela função de transferência de um filtro (selecionado conforme a aplicação).

Com isso, nessa seção é mostrada um pouco da teoria da transformada de Fourier, suas propriedades e aplicações em processamento de sinais e imagens e suas vantagens para comparar com a transformada quaterniônica de Fourier. A transformada de Fourier de uma função $f(x)$ real absolutamente integrável, denotada 
por $F(*)$ é definida para $w \in \mathbf{R}$, por (Oppenheim et al., 1998) $F(w)=\int_{-\infty}^{\infty} f(x) e^{-j w x} d x$.

Se $w=2 \pi w_{x}$, obtém-se a transformada de Fourier de $f(x)$ em função da frequência fundamental do sinal $w_{\bar{*}}$. Assim:

$$
F\left(w_{x}\right)=\int_{-\infty}^{\infty} f(x) e^{-2 \pi j x w_{w}} d x \text {. }
$$

Esta última pode ser estendida ao caso bidimensional:

$F\left(w_{z}, w_{y}\right)=\int_{-\infty}^{\infty} \int_{-\infty}^{\infty} f(x, y) e^{-2 \pi j\left(\pi w_{w}+y w_{y}\right)} d x d y$.

Segundo Sodré (2003), a transformada de Fourier de $f(x)$ é uma função $F(w)$ cuja imagem está no conjunto dos números complexos, logo esta pode ser decomposta em sua parte real e imaginária. Sendo $F_{r}(w)$ e $F_{i}(w)$, respectivamente, como as partes real e imaginária de $F(w)$ e $j=\sqrt{-1}$ (para não confundir com o índice $i$ ), temse:

$$
F=F_{r}+j F_{i}=|F| e^{j \theta} \text {. }
$$

Em que $\theta$ é o ângulo de fase da transformada de Fourier definido por $\theta(f)=\operatorname{arctg}\left(\frac{F_{i}}{F_{r}}\right)$.

A amplitude da transformada de Fourier $F(w)$ (ou espectro de amplitude do sinal $f(x))$ é definida como

$|F|=\sqrt{F_{r}^{2}+F_{i}^{2}}$.

A partir das definições da Transformada de Fourier pode-se definir seu espectro de potência $P(w)$ por

$$
P(w)=|F|^{2}=F_{r}^{2}+F_{i}^{2} \text {. }
$$

De acordo com Oppenheim (1998), a Transformada Inversa de Fourier de $g(w)$ é definida por:

$F^{-1}(g(w))=\frac{1}{2 \pi} \int_{-\infty}^{\infty} g(w) e^{j w t} d w$.

Se utilizar a mudança de variável $w=2 \pi f$ e substituir a diferencial $d w=2 \pi d f$, a função $F$ ficará dependendo da variável $f$. A definição ficará da forma $f(t)=F^{-1}(F(w))=\int_{-\infty}^{\infty} F(f) e^{2 \pi j t f} d f$. 
Isto garante a recuperação da própria função original através da aplicação da transformada inversa aplicada à transformada de Fourier, ou seja, $F^{-1}$ composta com $F$ é igual à identidade.

Substituindo a frequência $w^{w}$ por $w_{x}$, em que $w_{w}$ é a frequência que depende de $x$ na definição de Transformada Inversa de Fourier, pode-se obter outra forma para a Transformada Inversa de Fourier de $F\left(w_{x}\right)$, dada por $F^{-1}\left(F\left(w_{x}\right)\right)=\frac{1}{2 \pi} \int_{-\infty}^{\infty} F\left(w_{x}\right) e^{2 \pi j x w_{N}} d w_{x}$.

Dessa forma, pode-se estender a definição ao caso bidimensional $F^{-1}\left(F\left(w_{X}, w_{y}\right)\right)=\left(\frac{1}{2 \pi}\right)^{2} \int_{-\infty}^{\infty} \int_{-\infty}^{\infty} F\left(w_{X}, w_{y}\right) e^{2 \pi j(x, y) \cdot\left(w_{X} w_{y}\right)} d w_{x} d w_{y}$.

\subsection{Transformada discreta de fourier}

A transformada discreta de Fourier (DFT, Discrete Fourier Transform) é muito empregada no processamento de sinais e imagens bidimensionais, filtragem, realce, restauração, decodificação, etc. Seja uma sequência periódica bidimensional $f(x, y)=f(x+s M, y+r N)$, em que $M$ é o período das linhas, $N$ é o período das colunas e r.s são números inteiros que podem ser positivos ou negativos. A DFT é definida

por

$F(u, v)=\frac{1}{M N} \sum_{x=0}^{M-1} \sum_{y=0}^{N-1} f(x, y) e^{-2 \pi j\left(\frac{x x}{M}+\frac{w y}{N}\right)}$.

Sua inversa é dada por

$$
f(x, y)=\frac{1}{M N} \sum_{w=0}^{M-1} \sum_{v=0}^{N-1} F(u, v) e^{2 \pi j\left(\frac{M x}{M}+\frac{w y}{N}\right)} .
$$

Outra propriedade da transformada de Fourier diz que se $F(w)$ é a FT de um sinal real unidimensional $f: \mathbb{R} \rightarrow \mathbf{R}$, então $\overline{F(w)}=F(-w)$ (então $\overline{F(w)}$ é o conjugado de $F(w)), w \geq 0$, isto é, basta conhecer $F(w)$ para recuperar todo o sinal, ou seja, é necessário $50 \%$ da informação no domínio da frequência. Analogamente, seja $F$ a transformada de Fourier de um sinal real bidimensional $f: \mathbf{R}^{2} \rightarrow \mathbf{R}$, então $\overline{F\left(w_{1}, w_{2}\right)}=F\left(-w_{1},-w_{2}\right)$ e $\overline{F\left(-w_{1}, w_{2}\right)}=F\left(w_{1},-w_{2}\right)$, assim, é suficiente conhecer $F$ no

primeiro e no segundo quadrante do domínio da frequência para recuperar todo o 
sinal, ou seja, é necessário $50 \%$ da informação no domínio da frequência para restaurar $f$ (Pelaes e Santos, 2007).

\subsection{Quatérnios}

O matemático irlandês Sir Willian Rowan Hamilton (1805-1865) foi o primeiro) a compreender a importância de estender as propriedades aritméticas que caracterizam o corpo $\mathbf{R}^{2}$ dos números reais a conjunto de vetores. Seu primeiro passo foi introduzir um conjunto de vetores no $\mathbf{R}^{\mathbf{z}}$, o conjunto de pares ordenados de números reais. Este conjunto pode ser visto com um espaço vetorial de dimensão 2 sobre os reais, com base canônica $1=(1,0)$ e $i=(0,1)$. Com a soma usual e 0 produto entre esses vetores, esse espaço vetorial toma estrutura de corpo e sabemos que este corpo tem a mesma estrutura do corpo dos números complexos. Com base nesse conceito Hamilton teve a idéia de expandir a soma e o produto entre vetores do $\mathbf{R}^{2}$ para o $\mathbf{R}^{z}$ (espaço vetorial de dimensão três). Ele trabalhou neste caso, sem sucesso, durante o período de 1830 - 1843. Hamilton descobriu que era necessário considerar um espaço de quatro dimensões (Artmann, 1988). De fato, considerando no espaço vetorial $\mathbf{R}^{4}$ a base canônica $1, i, j, k$ podemos escrever um vetor $q \in \mathbf{R}^{4}$ como $q=a+b i+c j+d k$ em que $a, b, c, d \in \mathbf{R}$.

\subsubsection{Propriedades dos quatérnios}

Pode-se introduzir um produto em $\mathbf{R}^{\mathbf{4}}$, dado por

$i^{2}=j^{2}=k^{2}=i j k=-1$,

$i j=k, j k=i, k i=j$.

$j i=-k, k j=-i, i k=-j$.

Este produto estende-se, por linearidade, ao produto de quaisquer vetores $\mathbf{R}^{\mathbf{4}}$ e faz com que este produto ganhe uma estrutura algébrica em que valem todas as propriedades de corpo, exceto a propriedade da comutação, ou seja, o produto definido em $\mathbf{R}^{\mathbf{4}}$ não é comutativo. Tal estrutura é hoje denominada anel de divisão. $\mathrm{O}$ anel de divisão $\mathbf{R}^{\mathbf{4}}$ é denotado por $\mathrm{H}$ e chamado Anel dos Quatérnios de Hamilton (Lima, 2007). 
Um quatérnio $q=a+b i+c j+d k$ é constituído de uma parte escalar $E(q)=a$ e uma parte vetorial $V(q)=b i+c j+d k$. A parte vetorial do quatérnio é geralmente vista como um vetor em $\mathbf{R}^{\tilde{a}}$. Inclusive a notação $i_{, j}, k$ para os vetores da base canônica de $\mathbf{R}^{\text {a }}$, muito usada ainda hoje, foi criada por Hamilton para descrever os quatérnios. Segundo Artmann (1988), Hamilton definiu, também, o conjugado de um quatérnio $q=E(q)+V(q)$ como $\bar{q}=E(q)-V(q)=a-b i-c j-d k$

A norma e o módulo do quatérnio $q$ são definidos, respectivamente, por

$N(q)=q \bar{q}=a^{2}+b^{2}+c^{2}+d^{2}$ e $|q|=\sqrt{a^{2}+b^{2}+c^{2}+d^{2}}$.

Um quatérnio $q$ é dito unitário quando $N(q)=1$.

Deve-se observar a importância dos quatérnios, que em primeiro lugar, é historicamente o primeiro exemplo de uma estrutura algébrica não comutativa. Sendo que hoje que esse tipo de estrutura é abundante em Matemática. Além disso, com a construção dos quatérnios, Hamilton demonstra que é preciso lançar mão de espaços de dimensão superior a três, mesmo quando o objetivo é tratar problemas em $\mathbf{R}^{\mathbf{3}}$.

\subsubsection{Forma polar de um quatérnio}

Todo quatérnio $q=a+b i+c j+d k$ pode ser escrito como $q=|q|(\cos \varphi+e \cdot \operatorname{sen} \varphi), \quad 0 \leq \varphi \leq 2 \pi$,

em que $|q|$ é o módulo do quatérnio e $\varphi$ o seu argumento. Se $|q| \neq 0$, então

$\cos \varphi=\frac{a}{|q|} \mathrm{e}^{\operatorname{sen} \varphi= \pm \frac{\sqrt{b^{2}+c^{2}+d^{2}}}{|q|}}$

Tem-se também que e é um vetor unitário dado por $e= \pm \frac{b i+c j+d k}{\sqrt{b^{2}+c^{2}+d^{2}}}$

A fórmula de Euler para exponenciais complexas generaliza os quatérnios, ou seja, $q=|q| e^{\boxplus \varphi}$.

Veja que o vetor unitário $e$ é uma soma envolvendo as três componentes imaginárias, ou seja, pode-se escrever $e^{ \pm \varphi}$ como um produto (utilizando as propriedades das exponenciais). 
Assim, tomando-se $\phi= \pm \frac{b \varphi}{\sqrt{b^{2}+c^{2}+d^{2}}}, \quad \theta= \pm \frac{c \varphi}{\sqrt{b^{2}+c^{2}+d^{2}}} e^{\psi= \pm \frac{d \varphi}{\sqrt{b^{2}+c^{2}+d^{2}}}}$ obtém-se, outra forma de expressar, um quatérnios na sua forma polar como (Pelaes e Santos, 2007)

$q=|q| e^{i \phi} e^{\theta j} e^{\psi k}$ em que $\phi \in\left[-\pi, \pi\left[, \theta \in\left[-\frac{\pi}{2}, \frac{\pi}{2}\left[e^{\psi \in\left[-\frac{\pi}{4}, \frac{\pi}{4}\right.}[\right.\right.\right.\right.$.

\subsubsection{Forma simplificada se um quatérnio}

Segundo Ell e Sangwine (2007), a forma simplificada de um quatérnio começou com algumas idéias da forma de um quatérnio de Caley - Dickson, em que um quatérnio é definido recursivamente como um número complexo generalizado, cujas partes real e imaginária são números complexos. Os dois números complexos que formam tal número generalizado têm operadores diferentes e ortogonais.

Por exemplo, todo quatérnio $q=a+b i+c j+d k$ pode ser escrito na forma de Caley - Dickson como $q=A+B j$, em que $A=a+b i$ e $B=c+d i$. Observe que $i$ e $j$ são os operadores ortogonais.

Usando $\mu\left(\mu^{2}=-1\right)$ como operador complexo generalizado, tem-se a representação de um quatérnio na forma simplificada. Sejam $\mu_{1}$ e $\mu_{2}$ quatérnios puros imaginários, tais que $\mu_{1} \perp \mu_{z}$, um quatérnio arbitrário é representado na forma simplificada por

$q=A^{s}+B^{\mu_{\mu}}$.

Em que $A^{s}=a^{s}+b^{x} \mu_{1}$ e $B^{s}=c^{s}+d^{y} \mu_{1}$, então $q=\left(a^{s}+b^{x} \mu_{1}\right)+\left(c^{s}+d^{x} \mu_{1}\right) \mu_{2}$, ambos são isomórficos aos números complexos, uma vez que estão em um mesmo espaço complexo generalizado. Efetuando os produtos obtém-se $q=a^{y}+b^{s} \mu_{1}+c^{b} \mu_{\mathbf{2}}+d^{s} \mu_{3}$.

Em que $\mu_{3}=\mu_{1} \mu_{2}, \mu_{1} \perp \mu_{3}$ e $\mu_{3} \perp \mu_{2}$. Veja que 0 sistema de operadores $\mu_{1}, \mu_{2}, \mu_{3}$ é isomórfico ao sistema de operadores $i, j, k$. Essa forma de representação será usada adiante nas aplicações da Transformada Quaterniônica de Fourier. 


\subsection{Imagem colorida}

Segundo Sangwine e Ell (2000), uma imagem no sistema RGB pode ser expressa como um quatérnio imaginário puro, por exemplo, se $I(x, y)$ é a função que representa uma imagem então $I(x, y)=R(x, y) i+G(x, y) j+B(x, y) k$. Essas funções formam um espaço de cores e nesse espaço há vetores que caracterizam situações importantes para a análise de imagens, como por exemplo, o eixo de luminância (no espaço RGB esta é a linha cinza) representado pelo vetor (eixo) $\frac{i+j+k}{\sqrt{3}}$.

\subsection{Transformada quaterniônica de fourier}

Nesta seção será mostrada a QFT, suas propriedades e aplicações, comparando-as com a Transformada clássica de Fourier (FT, Fourier Transform).

Para Pelaes e Santos (2007), a transformada quaterniônica de Fourier (QFT, Quaternion Fourier Transform) é uma transformada bidimensional e (em geral) pode ser aplicada em funções quaterniônicas, (sobre certas condições, assim como a transformada de Fourier), porém consideram-se apenas sinais reais. A QFT é um caso particular das transformadas hipercomplexas de Fourier.

Seja $f$ um sinal bidimensional, $f: \mathbf{R}^{2} \rightarrow \mathbf{R}$, então a QFT de $f$ é definida por (Ell, 1993)

$F_{q}\left(w_{1}, w_{2}\right)=\int_{-\infty}^{\infty} \int_{-\infty}^{\infty} e^{-i x_{1} w_{1}} f\left(x_{1}, x_{2}\right) \cdot e^{-j x_{2} w_{2}} d x_{1} d x_{2} \cdot(23)$

Como a QFT é uma função quaterniônica, pode-se representar $F_{q}$ por

$F_{q}=F_{r}+i F_{i}+j F_{j}+k F_{k}$ (24) Em que $F_{r}$ é a parte real da transformada e $F_{1}, F_{j}, F_{k}$ são as componentes imaginárias e sua forma polar pode ser expressa por $A_{q} e^{i \phi} e^{j \theta} e^{k q \psi}$, em que $A_{q}$ é o espectro de amplitude do sinal. O espectro de potência do sinal $f(x)$ é definido por $P(w)=\left|F_{q}\right|^{2}=F_{x}^{2}+F_{i}^{2}+F_{j}^{2}+F_{k}^{2}$

.A Inversa da Transformada Quaterniônica de Fourier (IQFT, Inverse Quaternion Fourier Transform) é dada por (Pei et al., 2001)

$f(x, y)=\frac{1}{4 \pi^{2}} \int_{-\infty}^{\infty} \int_{-\infty}^{\infty} e^{i x_{1} w_{1}} F_{q}\left(u_{1}, w_{2}\right) \cdot e^{j x_{2} w_{2}} d u_{1} d w$.

Observe que para calcular a IQFT, é fundamental que $F_{q}$ esteja envolvida pelas exponenciais, nesta ordem, pois caso contrário $f$ não estaria definida. 


\subsubsection{Propriedades da QFT}

As propriedades de translação no tempo, translação na frequência, homotetia na variável e linearidade, todas aplicadas à transformada de Fourier, são aplicadas a QFT. Além dessas propriedades, a QFT tem outras propriedades importantes. Se $f$ é um sinal separável, ou seja, $f\left(x_{1}, x_{2}\right)=g\left(x_{1}\right) h\left(x_{2}\right)$ e $F_{q}=A_{q} e^{i \phi} e^{j \theta} e^{k \psi \psi}$, então $\psi=0$. Isto é, a QFT dependerá somente de dois fasores $\left(\phi \mathrm{e}^{\theta}\right) \mathrm{e}^{F_{\mathrm{q}}}=A_{q} e^{i \phi} e^{j \theta}$ (Pelaes e Santos, 2007). Segundo Bülow e Sommer (2001), existem três involuções não triviais definidas nos quatérnios. Essas involuções são dadas por $\alpha_{i}(q)=-i q i=a+b i-c j-d k, \alpha_{j}(q)=-j q j=a-b i+c j-d k, \alpha_{k}(q)=-k q k=a-b i-c j+d k(27)$ que são funções quaterniônicas. Outra propriedade dos quatérnios diz que uma função $f: \mathbf{R}^{2} \rightarrow \mathbf{R}$ é chamada Hermitiana Quaterniônica se $f\left(x_{1}, x_{2}\right)=\alpha_{j}\left(f\left(x_{1}, x_{2}\right)\right)$ e $f\left(x_{1},-x_{2}\right)=\alpha_{i}\left(f\left(x_{1}, x_{2}\right)\right)$. Da definição da QFT obtém-se que se $F_{q}$ é a QFT de $f$, então $F_{q}$ é Hermitiano Quaterniônico, ou seja, $F_{q}\left(-w_{1}, w_{2}\right)=-j\left(F_{q}\left(w_{1}, w_{2}\right)\right) j$ $F_{q}\left(w_{1},-w_{2}\right)=i\left(F_{q}\left(w_{1}, w_{2}\right)\right) i$.

\subsection{Transformada quaterniônica de fourier discreta}

A Transformada Quaterniônica de Fourier Discreta (DQFT, Discrete Quaternion Fourier Transform) de $f: \mathbf{R}^{2} \rightarrow \mathbf{R}$ e sua inversa são dadas por (Sangwine, 1996)

$$
\begin{aligned}
& F_{\mathrm{q}}(u, v)=S \sum_{X=0}^{M-1} \sum_{y=0}^{M-1} e^{-2 \pi j\left(\frac{M X}{M}\right)} f(x, y) e^{-2 \pi k\left(\frac{X}{N}\right)} . \\
& f(x, y)=S \sum_{\mathbb{W}=0}^{M-1} \sum_{\mathbb{N}=0}^{N-1} e^{2 \pi j\left(\frac{M}{M}\right)} F_{q}(u, v) e^{2 \pi k\left(\frac{W Y}{N}\right)} .
\end{aligned}
$$

Em que a função discreta $f(x, y)$ é de dimensão $M \times N$ e $S$ é o fator de escala $1 / \sqrt{M N}$. A ordem das exponenciais é importante, pois os quatérnios são não comutativos para a multiplicação (incluindo a multiplicação de um quatérnio por um número complexo usual). 


\section{METODOLOGIA}

Dada uma imagem na forma quaterniônica, $f(x, y)$, decomposta na forma simplificada dada por: $f(x, y)=f_{1}(x, y)+f_{2}(x, y) \mu_{2}$ (31) . Em que cada parte, $f_{1}$ e $f_{z}$, podem ser representadas como imagens complexas. Neste caso, cada uma das imagens quaterniônicas é equivalente a uma imagem complexa, que requer apenas duas componentes reais para a sua representação.

De acordo com Ell e Sangwine (2007), $\mu_{1}$ é o eixo ao longo da linha cinza e $\mu_{z}$ da linha vermelha, daí, $f_{1}$ (parte paralela) é a informação de luminância (esta ainda é uma imagem colorida, e não uma imagem em tons de cinza) e a parte $f_{\mathbf{z}} \mu_{\mathbf{z}}$ (parte perpendicular) é a informação de crominância. Essas duas partes podem ser somadas através da equação (31) para gerar a imagem original, como mostra a figura 1.

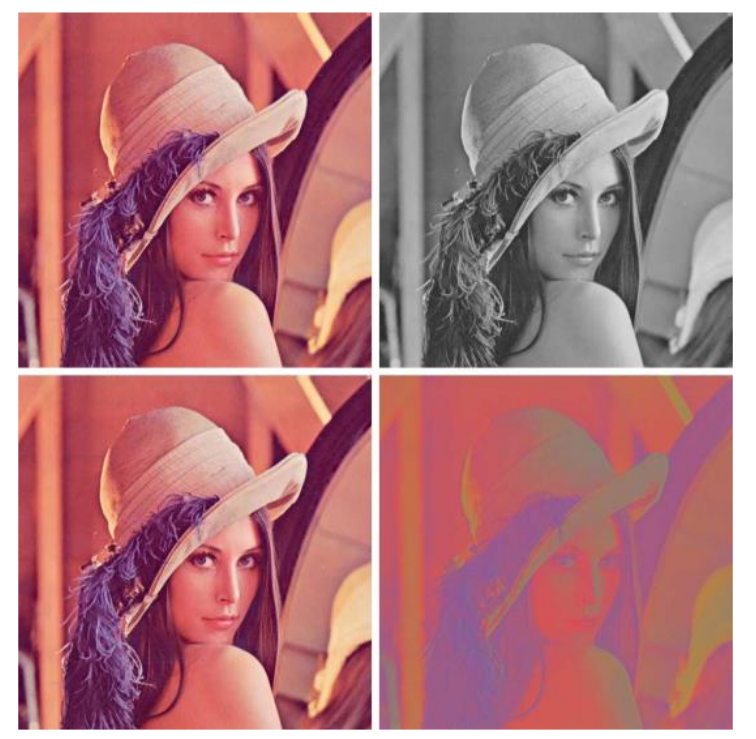

Figura 1: Decomposição simplificada da imagem de "Lena". No sentido horário a partir da superior: original, parte paralela, parte perpendicular e soma de ambas as partes. (Ell and Sangwine, 2007).

Dessa forma, é possível escrever a QFT na forma simplificada, como:

$F_{q}(u, v)=F_{1}(u, v)+F_{2}(u, v) \mu_{2}$ (32) Onde $F_{1}(u, v)$ é a parte paralela e $F_{2}(u, v) \mu_{\mathbf{2}}$ é a parte perpendicular. Ell e Sangwine (2007) desenvolveram um algoritmo para implementar a QFT. Segue, em resumo, os passos do algoritmo:

Decompor a imagem, $f(x, y)$, como $f(x, y)=f_{1}(x, y)+f_{2}(x, y) \mu_{2}$ em que cada $f_{i}$ pertence ao plano $\left(1, \mu_{1}\right)$; 
Expandir as componentes simplificadas como $f_{i}(x, y)=r_{i, 1}(x, y)+r_{i, 2}(x, y) \mu_{1} f_{i}(x, y)=r_{i, 1}(x, y)+r_{i, 2}(x, y) \mu_{1} \quad$ em que cada $r_{i, j}(x, y)$ é uma imagem de escalares;

Construir uma imagem complexa equivalente como $f_{i}^{u}(x, y)=r_{i, 1}(x, y)+r_{i, 2}(x, y) i$; Executar duas FFT bidimensionais de $f_{i}^{\prime}(x, y)$ para obter $F_{i}^{v}(u, v)=R_{i, 1}^{v}(u, v)+R_{i, 2}^{v}(u, v) i$;

Construir as componentes simplificadas como $F_{i}(u, v)=R_{i_{1}, 1}(u, v)+R_{i, 2}(u, v) \mu_{1}$; Construir a QFT na forma simplificada como $F_{q}(u, v)=F_{1}(u, v)+F_{\mathbf{z}}(u, v) \mu_{\mathbf{z}}$.

Observe que $\mu_{1}$ e $\mu_{2}$ são quatérnios unitários puros triviais, assim como $i$ e $j$, mas é possível utilizar um quatérnio unitário puro $(\mu)$ de forma generalizada de acordo com a imagem a ser tratada. De acordo com Sangwine (1996), a posição dos pontos no domínio espectral como uma função de frequência espacial horizontal e vertical são as mesmas na DQFT, assim como na DFT bidimensional. Ou seja, no formato usual de uma matriz bidimensional de pontos espectrais, as frequências mais altas são representadas mais próximas ao eixo horizontal ou vertical da matriz. Ao contrário da DFT bidimensional, a DQTF separa as quatro possíveis combinações de componentes cosseno e seno horizontais e verticais em quatro componentes espectrais quatérniônico. A DFT, em contrapartida, une essas componentes horizontais e verticais em um único componente real ou imaginário.

\section{RESULTADOS}

De acordo com a propriedade descrita pelas equações em (28), se $F_{q}$ for conhecida somente no primeiro quadrante, em que $w_{1} \geq 0$ e $w_{2} \geq 0$, então os valores dos demais quadrantes podem ser recuperados utilizando a propriedade descrita pela equação (28). $O$ quadrante $w_{1} \geq 0$ e $w_{2}<0$ pode ser reconstruído utilizando a equação $F_{q}\left(w_{1},-w_{2}\right)=i\left(F_{q}\left(w_{1}, w_{2}\right)\right) i$, o quadrante $w_{1}<0$ e $w_{2} \geq 0$ é obtido por $F_{q}\left(-w_{1}, w_{2}\right)=-j\left(F_{q}\left(w_{1}, w_{2}\right)\right) j$ e, por fim, o quadrante $w_{1}<0$ e $w_{2}<0$ é obtido por $\alpha_{i}\left(\alpha_{j}\left(F_{q}\left(w_{1}, w_{2}\right)\right)\right)$ que é igual a $\alpha_{i}\left(F_{q}\left(-w_{1}, w_{2}\right)\right)=F_{q}\left(-w_{1},-w_{2}\right)$. Isso mostra que para recuperar todo o sinal da QFT basta conhecer $25 \%$ do sinal no domínio da frequência (Bülow and Sommer, 2001). 
Outro resultado consiste na necessidade de obter a transformada de imagens coloridas holisticamente. Assim, considere um pixel de valor quaterniônico $a+b i+c j+d k$ multiplicado por dois exponenciais complexos de acordo com a definição da DQFT $(\cos \alpha-j \operatorname{sen} \alpha)(a+b i+c j+d k)(\cos \beta-k \operatorname{sen} \beta)$ em que $\alpha=\frac{2 \pi x u}{M} \mathrm{e}$ $\beta=\frac{2 \pi y v}{N}$ O resultado é um pixel de valor quaterniônico com as componentes cosseno e seno verticais e horizontais (Sangwine, 1997).

As equações (10) e (11) mostram que a DFT é semelhante à DQFT, a diferença é a ordem das exponenciais, que na DFT estão juntas, pois a álgebra dos números complexos é comutativa para a multiplicação. Com isso, considere um pixel de valor complexo multiplicado por dois exponenciais complexos de acordo com a definição da DFT $(a+b i)(\cos \alpha-i \operatorname{sen} \alpha)(\cos \beta-j \operatorname{sen} \beta)$, no qual $\alpha=\frac{2 \pi x u}{M} \mathrm{e}^{\beta=\frac{2 \pi y v}{N}}$. O resultado será um pixel de valor complexo contendo as combinações de cosseno e seno, como visto na DQFT.

De acordo com Sangwine (1997), as combinações de seno e cosseno aparecem nos resultados da DQFT e da DFT; a diferença é que, em ambos, essas combinações estão separadas em diferentes partes reais e imaginárias. Por exemplo, a combinação $\cos \alpha \cos \beta$ aparece na parte real do espectro da DFT com coeficiente $a$, e na parte imaginária com coeficiente $b$; e nas quatro partes do espectro DQFT com cada um dos coeficientes $a, b, c$ ou $d$. Assim, qualquer variação espacial de um determinado componente de cor a partir do conjunto $\{r, g$, b\} é separada em diferentes partes real ou imaginária do ponto espectral.

Significativamente, as informações de domínio espectral produzidas pela DQFT consistem de quatro números reais por ponto espectral (com a parte real zero, o sistema RGB é representado pelas três componentes imaginárias/vetoriais), ao invés das seis que seriam necessários para representar a Transformada de Fourier Complexa dos três componentes de cor separadamente. Poderia parecer que uma economia em armazenamento de dados pode ser feita usando o DQFT, e possivelmente uma compressão até melhor do que é conseguida com domínio da frequência de codificação de imagens. Os sinais são frequentemente discretos e, por isso, deve-se trabalhar com a DQFT como descrito nas equações (33) e (34) e assim implementar tal modelo. 
Segundo Pelaes e Santos (2007), a transformada clássica de Fourier tem sua representação discreta muito bem implementada pela famosa FFT (Transformada Rápida de Fourier, Fast Fourier Transform), cujo algoritmo tem complexidade o(NogN). Por essa razão a implentação da QFT deve ser feita usando a FFT, por isso FQFT (Fast Quaternion Fourier Transform). Para tanto, basta decompor as integrais da QFT de forma a se obter a transformada clássica de Fourier (ao fazer isso se deve tomar cuidado com a não comutatividade para a multiplicação dos quatérnios). Ao final, verifica-se que para calcular a FQFT de um sinal real é necessário calcular três vezes a FFT, enquanto usando a FFT2 (FFT bidimensional) é necessário aplicar duas vezes a FFT. Para a IFQFT é necessário quatro vezes a FFT, pois a IFQFT (Inverse Fast Quaternion Fourier Transform) é aplicada em uma função quaterniônica, enquanto que a IFFT2 utiliza duas vezes a IFFT. Assim, verifica-se que o acréscimo de no custo computacional é insignificante, já que se espera obter informações que a transformada de Fourier clássica não oferece.

\section{CONCLUSÕES}

Segundo Bas, Le Bihan e Chassery (2003), para projetar um esquema de imagem com cor eficiente deve-se encontrar um valor de $\mu$ tal que a percepção de impacto da componente paralela $F_{1}(u, v)$ seja mínima. Como $\mu$ deve ser unitário e a QFT é uma transformada ortogonal, o erro quadrático entre a imagem marcada (imagem em marca d'água) $I_{m}$ e a imagem original $I_{0}$ é igual ao erro quadrático entre a componente paralela da $F_{I_{m}}$ (QFT da imagem $I_{m}$ ) e a componente paralela da $F_{I_{0}}$ (QFT da imagem original).

Consequentemente, o erro médio quadrático entre $I_{m}$ e $I_{0}$ é independente de $\mu$. Foram feitos alguns experimentos para encontrar $\mu$ que minimize as distorções. Por exemplo, ${ }^{\mu_{\text {perc }}}={ }^{(-2 j+8 k)} / \sqrt{68}$ permite a construção de uma projeção que oferece interessantes propriedades de máscara para as diferentes imagens testadas. A figura 2 ilustra o impacto perceptivo da perturbação da parte paralela da $F_{I_{0}}$ com $\mu=\mu_{\text {pere }} \mathrm{e}^{\mu=\mu_{\text {Lum }}={ }^{(i+j+k)} / \sqrt{3}}$ com o mesmo erro quadrático médio (EQM) e erro absoluto médio (EAM). 

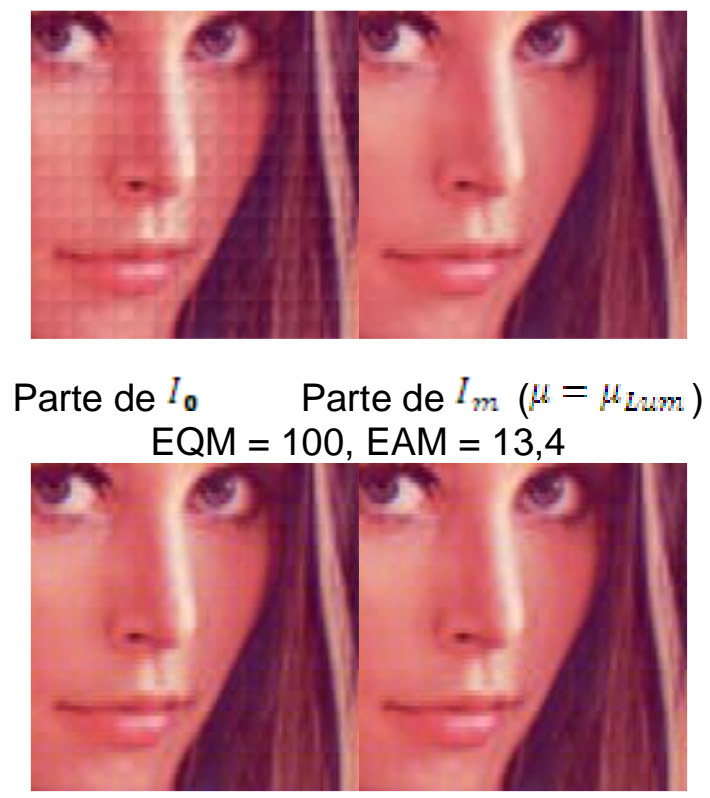

$$
\begin{array}{cc}
\text { Parte de } I_{m} & \left(\begin{array}{c}
\mu=\mu_{\text {pere }} \\
\mathrm{EAM}=13,4 \\
\mathrm{EQM}
\end{array}=100\right.
\end{array}
$$

Figura 2: Efeito de inserção de acordo com diferentes valores de $\mu$. (Bas et al., 2003).

Observe que os efeitos de máscara não dependem da cor média do bloco. Por exemplo, para $\mu_{\text {pere }}={ }^{(-2 j+8 k)} / \sqrt{68}$, o efeito de máscara é menor nas zonæ̧̧ azuis que em outras áreas coloridas. Assim pode encontrar $\mu$ em função de $\mu_{\text {pero }} \mathrm{e}$ $\mu_{i m}$, onde $\mu_{i m}$ representa um quatérnio unitário puro paralelo ao componente de cor da imagem:

Em que $E_{b}[X]$ representa a média do

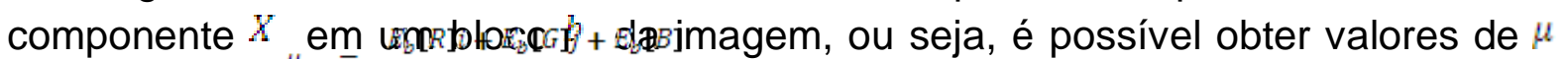

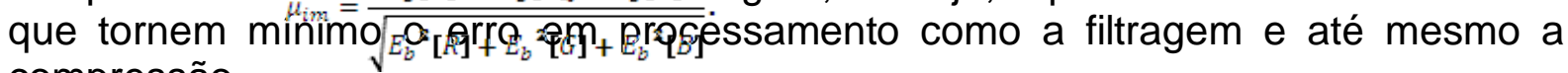
compressão.

\section{REFERÊNCIAS}

ARTMANN, B. (1988). The Concept of Number. Jonh Wiley \& Sons, Nova York.

BAS, P., Le Bihan, N. and Chassery, J. (2003). Color Image Watermarking Using Quaternion Fourier Transform. Laboratoire des Images et des Signaux INPG/CNRS.

BRAND, L. (1947). Vector and Tensor Analysis. Jonh Wiley \& Sons, Nova York.

BÜLOW, T. (1999). Hypercomplex Spectral Signal representations for the Processing and Analysis of Images. Institut Für Informatik und Praktische Mathematik, Bericht $\mathrm{N}^{\circ}$ 9903.

BÜLOW, T. and Sommer, G. (2001). Hypercomplex Signals - A Novel Extension of the Analytic Signal to the Multidimensional Case. IEEE Transations on Signal Processing, Vol. 49, N 11. 
ELL, T. A. (1993). Quaternion fourier transform for analysis of 2-dimensional linear time-invariant partial-differential systems. Proc. 32nd IEEE Conf. On Decision and Control.

ELL, T. A. and Sangwine, S. J. (2007). Hypercomplex Fourier Transform of Color Images. IEEE Transations on Image Processing, Vol 16, $\mathrm{N}^{\circ} 1$.

FELSBERG, M. and Sommer, G. (1999). Optimized Fast Algorithms for the Quaternionic Fourier Transform. Christian-Albrechts-University of Kiel, Institute of Computer Science and Applied Mathematics, Germany.

GONZALEZ, R. C. and Woods, R. E. (1993). Digital Image Processing. USA, AddisonWesley Pubisshing Company.

HAMILTON, W. R. (1853). Lectures on Quaternions. Hodges and Smith, Dublin.

LIMA, D. P. de O. (2007). Introdução aos Quatérnios. Monografia, CEFET-CE, Fortaleza.

MOXEY, C. E., Sangwine, S. J. and Ell, T. A. (2003). Hypercomplex Correlation Techniques for Vector Images. IEEE Transations on Signal Processing, Vol. 51, № 7.

NETO, J. F. (1999). Aplicação da Transformada de Fourier no Processamento Digital de Imagens, Universidade de Aracaju-Se.

OPPENHEIM, A. V., Schafer, R. W. and Buck, J. (1998). Discrete-Time Signal Processing, 2nd Edition, Prentice Hall, Upper Saddle River, New Jersey 07458.

PEI, S. C., Ding, J. J. and Chang, J. H. (2001). Efficient Implementation of Quaternion Fourier Transform, Convolution, and Correlation by 2-D Complex FFT, IEEE Transations on Signal Processing, Vol 49, № 11.

PELAES, D. e Santos, L. T. (2007). Processamento de Sinais Usando Quatérnios. Departamento de Matemática Aplicada. IMECC, UNICAMP.

SANGWINE, S. J. (1996). Fourier Transform of Colour Images Using Quaternion or Hypercomplex, Numbers. Electronics Letters, Vol. 32, № 21.

SANGWINE, S. J. (1997). The Discret Quaternion Fourier Transform. IEE Conference Publication $\mathrm{N}^{\circ} 443$.

SANGWINE, S. J. and Ell T. A. (2000). Colour Image Filters Based on Hypercomplex Convolution. IEE Proc.-Vis. Image Signal Process., Vol 147, № 2.

SANGWINE, S. J., Said, S. and Le Bihan, N. (2008). Fast complexified quaternion Fourier transform. Retirado de http://arxiv.org/abs/math.NA/0603578.

SHI, L. (2005). Explorations in Quaternion Colour. Simon Fraser University.

SODRÉ, U. (2003). Transformadas de Fourier, Curso de Ciências da Computação. 